\title{
Q. Pompeius Niger de Italica ${ }^{1}$
}

\author{
Luis Amela VALVERDE \\ Grupo CEIPAC. Universidad de Barcelona \\ amelavalverde@gmail.com
}

Recibido: 20 de febrero de 2011

Aceptado: 22 de marzo de 2011

\section{RESUMEN}

La aculturación de la onomástica por parte de los indígenas de Hispania es un fenómeno bien conocido. En este trabajo pretendemos analizar el tema de los «nombres de contacto», exactamente en el caso concreto de Q. Pompeius Niger, de Italica, que ha sido recientemente utilizado entre otros personajes para ejemplarizar este hecho. Exposición de esta teoría y comentario al respecto.

Palabras clave: Q. Pompeius Niger. Italica. Aculturación de la onomástica. Nomen Pompeius.

Amela Valverde, L., «Q. Pompeius Niger de Italica», Cuad. Fil. Clás. Estud. Lat. 31.1 (2011) 27-35.

\section{Q. Pompeius Niger of Italica}

\begin{abstract}
The acculturation of onomastics by the natives of Hispania is a well-known phenomenon. In this work we try to analyse the matter of the «names of contact», precisely in the specific one of Q. Pompeius Niger, from Italica, that has been recently used among other personalities in order to exemplify this fact. Exposition of this theory and commentary about it.
\end{abstract}

Keywords: Q. Pompeius Niger. Italica. Acculturation of onomastics. Nomen Pompeius.

Amela Valverde, L., «Q. Pompeius Niger of Italica», Cuad. Fil. Clás. Estud. Lat. 31.1 (2011) 27-35.

\footnotetext{
${ }^{1}$ Este trabajo se ha realizado en el marco del Proyecto I+D+I2009-2011 HAR2008-00210.
} 
De nuestras lecturas, ya antes había conocido la existencia de los «nombres de contacto», es decir, de antropónimos que pueden explicarse en dos (o más) diferentes lenguas a la vez (al. «Decknamen», ingl. «cover names», fr. «noms d'apparence latine»). Lo que nunca nos hubiéramos podido imaginar es que el gentilicio Pompeius, al cual hemos dedicado varios estudios (Amela 2001; 2002; 2004; 2005; 2007; 2008a; 2008b; 2009a; 2009b), pudiera ser uno de ellos.

Zeidler (2005, pp.175-200), en un estudio sobre los «amici» ${ }^{2}$ conocidos por las fuentes literarias de origen hispano de finales de la República, llega a la conclusión que todos ellos llevan un nombre que se puede explicar a través de las lenguas indígenas, aunque tengan una apariencia romana y se encuentren en la Italia central (Zeidler 2005, p.193). Si bien la idea es atrayente, consideramos que es un tanto excesiva, debido a que generalmente se considera que hubo una importante emigración itálica a la Bética (Marín 1988), de donde provienen la mayoría de nombres estudiados por Zeidler ${ }^{3}$.

De esta manera, nos ha llamado mucho la atención la inclusión de Q. Pompeius Niger entre los «nombres de contacto» (Zeidler 2005, pp.188-189). Este personaje aparece mencionado en el Bellum Hispaniense, relato que cuenta la campaña de C. Julio César (cos. I 59 a.C.) en la Hispania Ulterior contra los hijos de Cn. Pompeyo Magno (cos. I 70 a.C.), que desembocó en la batalla de Munda (45 a.C.). De esta manera es descrito: Q. Pompeius Niger eques Romanus Italicenses (BHisp.25.4). Por tanto, se da sus tria nomina, condición social, y su lugar de origen, Italica (Santiponce, prov. Sevilla). Se da la circunstancia de que durante esta campaña militó en el bando cesariano.

Zeidler señala la teoría de Dyson de que la dispersión de diversos nomina en Hispania, como, p.e., los Iunii, Licinii o Pompeii (Dyson 1980-1981, pp.288-289), están relacionados con las actividades de miembros de estas grandes familias romanas. Si bien reconoce que ejercieron una considerable influencia en las poblaciones locales, no puede responder a todas las peculiaridades registradas. De hecho, Zeidler señala dos problemas en relación a la tesis defendida por Dyson: primero, muchos nombres hispánicos no se encuentran entre las familias influyentes (p.e., Balbus entre los Cornelii); segundo, la distribución de los nombres no siempre sigue las actividades de estos prominentes romanos (p.e., los mismos Pompeii [Dyson 1980-1981, p.289] ${ }^{4}$ ). En consecuencia, forzosamente se ha de buscar otra explicación (Zeidler 2005, pp.194-195).

Debido a que la asunción de los dua o tria nomina no siempre significa la tenencia de la ciudadanía romana (como ejemplifica el caso de los jinetes hispánicos del

\footnotetext{
${ }^{2}$ El trabajo forma parte del proyecto «Amici Populi Romani. Prosopographie der auswärtiger Freunds Roms», dirigida por A. Coşkun en la Universidad de Tréveris.

${ }^{3}$ Zeidler (2005, p.199) considera que de los 25 personajes estudiados en su trabajo, sólo 5 (y eso que pone a uno en duda), es decir, un $20 \%$, no pueden ser recordados como asonante o semánticamente relacionados con las tradiciones onomásticas indígenas.

${ }^{4}$ Zeidler (2005, p.188) señala que si bien el renombre de Pompeii romanos puede haber influenciado en la selección por los indígenas de los nombres a finales del periodo republicano, un motivo onomástico nativo puede haber influido.
} 
Bronce de Ascoli [CIL I ${ }^{2} 709=$ CIL VI $37045=$ ILLRP $515=$ ILS 8888], con el añadido que ninguno de ellos lleva el nomen Pompeius), esto implicaría que la selección de antropónimos por los peregrini está menos sujeto a la influencia de patroni romanos de lo que se había asumido (Zeidler 2005, p.195).

De este modo, Zeidler (2005, pp.188-189) nos indica que el gentilicio Pompeius y nombres similares ${ }^{5}$ son generalmente asociados con el osco *pompe, «cinco» (Lejeune 1976, p.69; Untermann 2000, p.604); también ocurre en el etrusco Pumpna/Pun$p a n a^{6}$. Weisgerber (1968, pp.182, 212 y 233) ya señaló la frecuencia de Pompeius en las zonas de habla céltica y supuso una conexión con antropónimos galos con el numeral pempe, «cinco»; cf. también el praenomen Quintus o Alföldy $(1969, \mathrm{p} .111)$ en la diseminación de nombres con Pomp-. Hay una asonancia con el galo pempe «cinco» (Delamarre, 2003, 248), que es p.e. conocido de pempe-dula (Dioscurides 4, 42: $\pi \in \nu T a ́ \phi v \lambda \lambda$ ov, Ps.-Apuleyo 2,32 quinquefollium). Delamarre refiere a Pentius, Pentis, Pentilius, etc., que puede reflejar una forma reducida de *pempto-, «quinto» ${ }^{7}$. En una inscripción céltica ${ }^{8}$ de Oderzo (Od. 7) (Eska y Wallace 1999, pp.123-136), una forma pompe con $-O$ - asimilada (como en antiguo irlandés cóic) aparece en pompete(n)guaios «(el que conoce) cinco lenguas».

Así mismo, kuini- es la forma para «cinco» en celtíbero (Wodkto 2000, p.209), con esperada $k^{w}$ en lugar del galo/lepóntico $p$. P.e., las palabras en latín y britónico en $p$ - eran, generalmente, alteradas a $q$-, posteriormente a $c$-, cuando es prestado al irlandés (es decir, latín presbyter, primitivo galés *primiter se convirtió en irlandés ogámico QRIMITIR) (Zeidler 2005, pp.188-189).

Por otra parte, el nombre Niger está frecuentemente atestiguado en la Galia Narbonense y figura tanto entre ciudadanos romanos como en peregrini (Christol 2001, p.31). Ha sido clasificado como un nombre de contacto por Rémy (2001, p.105) en su estudio onomástico del Viennois, y lo justifica. Probablemente es una traducción del céltico dubu-/dubi-/dubno-, «oscuro, negro» (Delamarre 2003, pp.151-153). En Hispania, está frecuentemente atestiguado (Abascal 1994, p.439), y puede también relacionarse con el ausco-aquitano (vasco) belz, «negro», que puede estar asociado al ibérico bels ${ }^{9}$. En ambas lenguas, bels/beltz es frecuente en nombres propios, que se utilizaron hasta la Edad Media.

El praenomen Quintus, nos dice Zeitler (2005, p.189), es la causa por la que se ha relacionado a Q. Pompeius Niger con el homónimo cónsul del año 141 a.C. Quintus es un praenomen común, y puede haber sido seleccionado arbitrariamente como una parte necesaria de los tria nomina. Sin embargo, dentro del contexto de la aculturación onomástica, hay que señalar que es muy similar a nombres célticos comunes. En celtíbero, kuint- (para $\left.* k^{w} i n \chi t-?\right)$, «quinto», es casi idéntico al número ordi-

\footnotetext{
${ }^{5}$ Coşkun (2003, p.44) señala que Pomponius podría tener un origen céltico, al menos en la Galia.

6 Índices de Rix (1991, pp.155-156).- Duchesne (1934, p.89) consideraba que Pompeius era de origen etrusco.

7 Índice de casos en Hispania en Abascal (1995, pp.453-454).

${ }^{8}$ Que anteriormente había sido considera como véneta.

9 Índices de Untermann (1990, pp.216-217).
} 
nal latino. De hecho, Untermann (1996, p.144) ya observó que Quint- se concentraba en Celtiberia y en territorio de los Vettones, y podía indicar un nombre de contacto. Adicionalmente, los nombres Quint- muestran una distribución complementaria a los nombres Pent-/Pint-, que se dan en Asturias y Cantabria, y que tendrían el mismo significado (Untermann 1996, p.144). Luego Quintus Pompeius podría ser un juego de palabras con el motivo «cinco» en el contexto del latín y/o lenguas nativas.

En definitiva, las palabras Quintus, Pompeius y Niger son la traducción de palabras indígenas, siendo además que el praenomen es así mismo una adaptación morfológica (Zeitler 2005, p.195). Es decir, que Pompeius es un nombre de contacto. Sea como fuere, el propio Zeitler (2005, p.197) indica que algunos de los nombres pueden ser ambiguos, ya que recuerda que Italica fue fundada en el año 206 a.C. por P. Cornelio Escipión Africano (cos. I 205 a.C.) y poblada con veteranos tras la batalla de Ilipa (App. Ib. 38), y que si bien Niger puede tener una base indígena, también es un apodo muy difundido en la Italia central.

Igualmente, Zeidler nos señala que una posibilidad más lejana de explicación es una asonancia con la base onomástica cintu-, con sentido de «primero» (Meissner 2004, pp.97-100), p.e. Centus, Cintus, Cintonnus, Cintugenus, Cintugnatus, Cintullus, Cintusmus, Cintusmina, etc. (Delamarre 2003, p.117), Estos nombres son frecuentemente recordados en la Galia, junto con sus equivalentes latinos con base prim-, como: Primus, Primius, Primio, Primulus, Primitius, etc. Salomies (1987, p.115) puntualiza que Primus estaba muy difundido en Italia septentrional y áreas vecinas, pero en el resto del Imperio Romano es muy raro, porque habría sido exportado a estas últimas regiones.

Sobre nuestro personaje, González Román (1986-1987, p.72; 1994, p.294; 2001, p.180; 2005, p.304) señala que el gentilicio latino Pompeius tiene una amplia difusión por la península italiana (Conway 1967, p.580) y en la onomástica hispana (CIL II, 1069-1070; Knapp 1978, pp.212-214; Abascal 1994, pp.198-201; Lörincz 2000, pp.149-151; Amela 2003, pp.308-314 y 2004, pp.94-105), Si bien señala que en los Fasti Hispanienses se documentan diversos Pompeii como magistrados cum imperio, prefiere señalar la presencia de Q. Pompeius Niger en Hispania en el contexto de la emigración itálica.

Aquí surge el primer desconcierto, ya que Zeidler (2005, p.188) señala que no está claro de dónde $Q$. Pompeius Niger obtuvo la ciudadanía romana (lógicamente, para él se trata inicialmente de un indígena peregrino), si de Q. Pompeius (cos. 141 a.C.) o de Cn. Pompeyo Magno (cos. I 70 a.C.), y menciona como fuentes de este último aserto a González Román y Marín Díaz (1994, p.72). Pero estos últimos nada dicen sobre ello sino que, como hemos mencionado, lo consideran producto de la emigración itálica a la Península Ibérica; así mismo, también cita como autoridad a Weinrib (1990, pp.59ss), pero consultado este último autor, nada dice sobre este particular. Con ello, Zeidler es el único estudioso que cita a $Q$. Pompeius Niger como un indígena.

Igualmente, en su estudio, Zeidler (2005, p.198) no trata a todos los amici hispánicos, ya que se deja un total de diez, algunos de los cuales, como él mismo indica, no serían hispanos, pero alguno de ellos quizás pondría en apuros su teoría como, 
por ejemplo: C. Flavius, L. Munatius Flaccus, Calpurnius Silvanus o Annius Scapula. Así mismo, si se compara el origen que da Zeidler a ciertos personajes con los que da González Román, vemos que en el primero les da un origen nativo mientras que el segundo uno de carácter emigrante; no entramos en los detalles porque el caso de Q. Pompeius Niger es paradigmático.

Por nuestra parte, hemos defendido en otros trabajos que la aparición del nomen Pompeius en Hispania puede deberse a tres causas fundamentales ${ }^{10}$ : concesión de la ciudadanía romana por parte de Pompeyo Magno o sus hijos (p.e., los Pompeii de Celsa); aculturación de la onomástica por parte de los indígenas, fuesen o no clientes de la gens Pompeia (los Pompeii del Noroeste peninsular); emigración de itálicos ${ }^{11}$. Precisamente, Q. Pompeius Niger lo poníamos nosotros como ejemplo evidente de un emigrante itálico (o su descendiente) con este nombre (Amela, 2001, p.255; 2003, p.58 n.94 y pp.293-296; 2004, pp.57-58), sin tener relación alguna con los famosos Pompeii Magni.

Que hubo Pompeii emigrantes está probado por dos casos provenientes de la Galia Narbonense. Así, en primer lugar, tenemos a A. Pompeius A. f. Sab(atina) Pius (AE 1988 859), documentado en Arelate en el s. I d.C., cuya onomástica señala su proveniencia del norte-nordeste de Etruria, tanto por su cognomen como por su tribu, a lo que hay que añadir el nombre de su madre, Kareia Ingenua, cuyo gentilicio también es propio de esta región italiana (Dondin-Payre 1992, pp.81 y 83-84). Igualmente, T. Pompeius T.f. Trom. Albinus, de Vienna (CIL XII 2327), así mismo del s. I d.C., es de ascendencia italiana, ya que su tribu no se encuentra en la Galia (Rémy y Ferbier 1991, p.70).

En cuanto a la ciudad de Italica, el establecimiento de Cornelio Escipión no fue ex novo, sino que se realizó sobre un oppidum turdetano fundado en el s. IV a.C. y, como muestran los restos arqueológicos, nativos e inmigrantes convivieron en esta ciudad (González Román 2001, p.311). En época del emperador Augusto era municipium civium Romanorum, como documentan las monedas (RPC 60-63), hasta que en tiempos de Adriano fue elevada a la categoría de colonia (Gell. 16, 13, 4).

Evidentemente, habría indígenas habitando en Italica, pero es muy difícil considerar a $Q$. Pompeius Niger como uno de ellos. Sobre este asunto, hay que recordar que Apiano, al describir los acontecimientos bélicos del año 143 a.C., nos informa que el gobernador de la Hispania Ulterior, al retirarse a los cuarteles de invierno en la ciudad de Corduba, dejó el mando de las tropas a Q. Marcius,

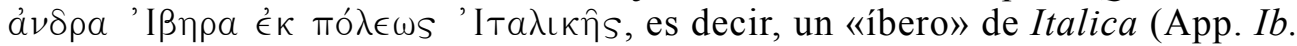
66). Por este motivo, se ha considerado al personaje citado por el escritor alejandrino como un nativo romanizado (García y Bellido 1960, p.60), aunque también un emigrante o descendiente de emigrantes (González Román 1994, p.283).

Caballos $(1994$, p.264; 2006, p.591) considera que C. Marcius sería un descendiente en tercera generación de los soldados asentados en Italica por Cornelio

\footnotetext{
${ }^{10}$ Una cuarta posibilidad pudiera ser que, un cliente de Pompeyo Magno, al que éste concedió la ciudadanía, se estableciera en Hispania, aunque no se tiene documentado testimonio alguno.

${ }^{11}$ Sobre onomástica y aculturación en la Hispania meridional, Vid: González Román (2001, pp.174-178).
} 
Escipión. Su razonamiento es claro: sería ilógico poner al mando del ejército romano, que estaba sufriendo derrota tras derrota frente al caudillo lusitano Viriato, a un indígena que recientemente habría adquirido el derecho de ciudadanía, hecho singular que considera este autor habría quedado reflejado en las fuentes; así mismo, la inexistencia de un magistrado cum imperio en Hispania con este gentilicio impide dar la responsabilidad a alguien de la concesión de este privilegio a nuestro personaje.

En realidad, el primer argumento de Caballos es el que consideramos más viable, puesto que C. Marcius, si fuese un indígena romanizado, podría haber tomado su nombre de un emigrante cualquiera, como acontece con los jinetes del Bronce de Ascoli. De esta forma, despejamos la posibilidad de que este individuo se tratara de un indígena, y fuese utilizado como argumento para esgrimir que $Q$. Pompeius Niger también lo era.

Por tanto, podemos concluir, según nuestro punto de vista, que $Q$. Pompeius $\mathrm{Ni}$ ger era realmente un descendiente de emigrantes, fuese de los instalados por Cornelio Escipión en el año 206 a.C. o en un momento posterior. Esto no invalida la posibilidad de que, efectivamente, algunos de los individuos con el nomen Pompeius que se conocen a través del registro epigráfico en Hispania habrían adoptado este gentilicio por parecerse a su onomástica, es decir, un «nombre de contacto».

De hecho, nosotros defendemos que la mayor parte de los Pompeii hispánicos tienen este nombre debido a una aculturización de su onomástica (mimetismo, como lo denomina Gómez-Pantoja [2003, p.251]), en relación o no con Pompeyo Magno, aunque sin duda la importancia de este último en la Historia podría haber influido en la utilización por los indígenas de este gentilicio (Amela 2001, p.259; 2003, p.307; 2004, p.88; 2005, p.175. 2008a, p.462; 2008b, p.141).

Casos, como, p.e., Pompeius Docilico(n) de San Esteban de Gormaz (prov. Soria) (AE $1995875=$ CIL II $2816=$ HEp 6 891), L. Pompeius Custumus Ammonis $f$. de Clunia (CIL II $2797=$ CIRPB $101=$ ERClunia $223=$ HEpOL $8623=$ ILER 2489), L. Pompeius Fuscinus Langiocum Fuscif. de Malamoneda (prov. Toledo) (CIL II $3088=$ HEp $160=$ HEpOL 199), C. Pompeius Gal. Caturonis f. (Mot?)ugenus de Uxama (CIL II $2403=$ CIL II $5557=$ CIL II $5753=$ CLE $876=$ ERSoria 165 $=$ HEpOL $8227=$ ILS 4514a), Pompeia Flavina Flavif. de Poza de la Sal (prov. Burgos) (AE $1976318=$ CIRPB 517), Pompei (a) Flac. f. Nementina de Yanguas (prov. Soria) (ACER R86 = ERSoria $36=$ HEp $5756=$ HEp 10 593) son, para nosotros, evidencia de que los indígenas utilizaron este nomen como un elemento de la romanización de su onomástica (nótese que ninguno de ellos utiliza los praenomina típicos de los Pompeyos Magnos, Cnaeus y Sextus), como muy posiblemente los Pompeii hispánicos que tenían cognomina prerromanos. El testimonio de Macrio Pompeiae $f$. de Torre de Santa María (prov. Cáceres) (CPCác. $497=$ HAE $939=$ HEpOL 25489 = ILER $2734=$ IRPC I 355) va en este mismo sentido.

Para finalizar, queremos llamar la atención de la existencia de un Pumpeius o Punpeius (= Pompeius) en una inscripción en escritura latina (CISP KENFG/1/2) procedente de Eglwys Nynnydd, Kenfig (Glamorganshire, Gales), del s. VI d.C., y, en la misma piedra, figura un epígrafe en escritura ogámica en el que se menciona a un $\mathrm{Po}$ - 
pia(s) o (también leído Pampes) ${ }^{12}$ (CISP KENFG 1/1), forma celtizada de Pompeius (Rhys 1899, p.132. RCAHMW, 1976, p.38). Evidentemente, no se puede buscar a un cliente de Pompeyo Magno en la «Dark Age» de Britania, ni tampoco creemos que se trate de un descendiente de uno de ellos, sino que es una prueba de que los indígenas utilizaban nombres latinos para homologarse a la cultura dominante.

\section{REFERENCIAS BIBLIOGRÁFICAS}

Abascal Palazón, J.M. (1994), Los nombres personales en las inscripciones latinas de Hispania, Murcia, Universidad de Murcia.

Abascal Palazón, J.M. y Espinosa, U. (1989), La ciudad hispano-romana. Privilegio y poder, Logroño, Colegio Oficial de Aparejadores y Arquitectos Técnicos de La Rioja.

AlföLdy, G. (1969), Die Personennamen in der römischen Provinz Dalmatia, Heidelberg, Carl Winter.

Amela Valverde, L. (2001), «El nomen Pompeius en Hispania: Algunos aspectos críticos», Emerita 69, 241-262.

Amela Valverde, L. (2003), Las clientelas de Cneo Pompeyo Magno en Hispania, Barcelona, Universidad de Barcelona.

Amela Valverde, L. (2004), «Las concesiones de ciudadanía romana: Pompeyo Magno e Hispania», $A C$ 73, 47-107.

Amela Valverde, L. (2005), «Los Pompeii de Hispania en época de los Antoninos. El caso de la Celtiberia», en Hernández Guerra, L. (ed.), Actas del II Congreso Internacional de Historia Antigua. La Hispania de los Antoninos (98-100), Valladolid, Universidad de Valladolid, pp.167-177

Amela Valverde, L. (2008a), «Los Pompeii de la actual provincia de Soria», Celtiberia 102, $445-468$

Amela Valverde, L. (2008b), «Los Pompeii de Clunia», HAnt 32, 131-142.

Amela VAlVerde, L. (2009a), «Un problema práctico en la epigrafía: la resolución de las abreviaturas en los nombres personales. El caso de los Pompeii hispánicos», Documenta \& Instrumenta 7, 105-121.

Amela ValVerde, L. (2009b), «Sobre testimonios tardíos del nomen Pompeius en Hispania», Fortunatae 20, 9-16.

BALIL, A. (1955-1956): «Algunos aspectos del proceso de la romanización de Cataluña», Ampurias 17-18, 39-57.

Brogan, O. (1953): Roman Gaul, Cambridge, G. Bell and Sons Ltd.

Caballos Rufino, A. (1989), «Los senadores de origen hispano durante la república romana», en GonZÁlez, J. (ed.), Estudios sobre Urso. Colonia Iulia Genetiva, Sevilla, Alfar, pp.233-279.

Caballos Rufino, A. (2006), s.u. «Marcius», Diccionario Akal de la Antigüedad Hispana, Madrid, Akal.

Christol, M. (2001), «Épigraphie et onomastique dans la cité de Nîmes du milieu du Ier s. av. J.-C. à la seconde moitié du Ier s. ap. J.-C.: analyse d'un échantillon», en DonDIN-

${ }^{12} \mathrm{Si}$ no es que la lectura de la inscripción permitiera leer el genitivo del antropónimo Pompeius. 
Payre, M. - Raepsaet-Charlier, M.T. (eds.), Noms, Identités culturelles et Romanisation sous le Haut-Empire, Bruxelles, Le Livre Timperman, pp.17-38.

CoşKun, A. (con contribuciones de J. ZEIDLER) (2003), 'Cover names' and Nomenclature in Late Roman Gaul. The Evidence of the bordelaise Poet Ausonius, Oxford, Oxford University Press.

Delamarre, X. $\left(2003^{2}\right)$, Dictionnaire de la langue gauloise. Une approche linguistique du vieux-celtique continental, Paris, Errance.

Dondin-Payre, M. (1992), «Une table en bronze inscrite d'Arles. Hommages publics et pratiques testamentaires chez les notables d'ascendance italienne», Gallia 42, 81-87.

DuChesne, J. (1934), «Note sur le nom de Pompée», AC 3, 81-89.

Dyson, St.L. (1980-1981), «The Distribution of Roman Republican family Names in the Iberian peninsula», AncSoc 11-12, 257-299.

Eska, J.F. - Wallace, R.E. (1999), «The Linguistic Milieu of *Oderzo 7», Historische Sprachwissenschaft 112, 123-136.

García y Bellido, A. (1960), Itálica. Colonia Aelia Augusta, Sevilla, C.S.I.C.

GARCÍA Moreno, L.A. (1987): «Presupuestos ideológicos de la actuación de Roma durante el proceso de la conquista de Hispania», Gerión 5, 211-243.

Gómez-Pantoja, J. (2003), «Ex ultima Celtiberia: Desarrollo municipal y promoción social en las viejas ciudades arévacas», en CASTILlo GARCíA, C. - RodrígUEZ NeILA, J.F. - JAVIER NaVArro, F. (eds.), Sociedad y economía en el Occidente romano, Pamplona, EUNSA, pp.231-282.

GonzÁlez Román, C. (1986-1987), «La onomástica del «Corpus» cesariano y la sociedad de la Hispania meridional», SHHA 4-5, 65-77.

GonzÁlez RomÁn, C. (1990), «Deditici y clientes en el área ibérica de la Hispania republicana con anterioridad a las Guerras Civiles», en AA.VV., Esclavos y semilibres en la Antigüedad clásica, Madrid, Universidad Complutense de Madrid, pp.187-205.

GonzÁlez RomÁn, C. (2001), «Prosopografía y romanización de las elites; a propósito de la Hispania meridional en época republicana», en LóPEz CASTRO, J.L. (ed.), Colonos y comerciantes en el occidente mediterráneo, Almería, Universidad de Almería, pp.171-188.

GonzÁlez RomÁn, C. (2002), «La expansión de la ciudadanía romana en la Bética», SCO 48, 307-339.

GonzÁlez Román, C. (2005), «Prosopografía del Bellum Hispaniense», en Melchor GiL, E. - Mellado Rodríguez, J. - Rodríguez-NeIla, J.F. (eds.), Julio César y Corduba: tiempo y espacio en la campaña de Munda (49-45 a.C.), Córdoba, Servicio de Publicaciones de la Universidad de Córdoba, pp.311-360.

GonzÁlez RomÁn, C. - MARín DíAz, M.A. (1994), «Prosopografía de la Hispania Meridional en época republicana», en GonzÁlez RomÁn, C. (ed.), La sociedad en la Bética. Contribuciones para su estudio, Granada, Universidad de Granada, pp.241-318.

KNAPP, R.C. (1977), Aspects of the roman experience in Iberia 206-100 B.C., Valladolid, Universidad de Valladolid.

KNAPP, R.C. (1978), «The Origins of Provincial Prosopography in the West», AncSoc 9.187-222.

LeJeune, M. (1976), L'anthroponymie osque, Paris, Les Belles Lettres.

LÖRINCZ, B. (composuit et correxit) (2000), Onomasticon Provinciarum Europae Latinarum. Vol. III: Labareus-Pythea, Wien, Forschungsgesellschaft Wiener Stadtarchaäologie.

MARín DíAz, M.A. (1986-1987), «La emigración itálica a Hispania en el siglo II a.C.», SHHA 4-5, 56-63. 
MeIsSNER, T. (2004), «Zum gallischen Zahlwort für 'erster' - ein indirekter Zeuge?», Historische Sprachforschung 117, 97-100.

Montenegro, A. (1986), «El régimen administrativo romano y la evolución de las organizaciones políticas indígenas», en A. MonTENEGRo DuQue - J.M. BlÁZQUEZ MARTínEZ - J.M. Solana SAInZ, Historia de España 3. España romana, Madrid, Gredos, pp.161-210.

Ooteghem, J. van (1954), Pompée le Grand, bâtisseur d'empire, Bruxelles, Palais des Academies.

POKORNY, J. (19725), Indogermanisches etymologisches Wörterbuch, 2 vols., Bern, Francke Verlag.

RÉmY, B. (2001), «La denomination des Viennois à l'époque imperial», en Dondin-PAYRE, M. - RAepsAet-Charlier, M.T. (eds.), Noms, Identités culturelles et Romanisation sous le Haut-Empire, Bruxelles, Le Livre Timperman, pp.55-174.

RCAHMW [Royal Commission on Ancient and Historical Monuments in Wales] (1976), An Inventory of the Ancient Monuments in Glamorgan. 2 vols., Cardiff, HMSO.

RÉmy, B. - Ferbier, E. (1991), «Une inscription de la cité de Vienne retrouvée (CIL XII 2327)», RAN 24, 267-270.

RHYs, J. (1899) «Some Glamorgan Inscriptions», Archaeologia Cambrensis 54.132-168.

Rix, H. (1991), Etruskische Texte. Ed. min., vol. 1: Einleitung, Konkordant, Indices, Tübingen, Narr.

SAlomies, O. (1987), Die römischen Vornamen. Studien zur römischen Namengebung, Helsinki, Societas Scientiarum Finnica.

Syme, R. (1958), Tacitus. 2 vols., Oxford, Clarendon Press.

Thouvenot, R. (1940), Essai sur la province romaine de Bétique, Paris, E. de Boccard.

Tranoy, A. (1981), La Galice Romaine. Recherches sur le nord-ouest de la Péninsule Ibérique dans l'Antiquité, Paris, Diffusion de Boccard

Tsirkin, Ju. B. (1989), «The Veterans and the Romanization of Spain», Gerión 7.137-147.

Untermann, J. (1990), Monumenta linguarum hispanicarum vol. 3. Die iberischen Inschriften aus Spanien, Wiesbaden, Ludwig Reichert.

Untermann, J. (1996), «VI. Onomastics», en Beltrán, F. - De Hoz, J. - Untermann, J., El tercer bronce de Botorrita (Contrebia Belaisca), Zaragoza, Gobierno de Aragón, pp.109166.

Untermann, J. (2000), Wörterbuch des Oskisch-Umbrischen, Heidelberg, Universitätsverlag C. Winter.

WeInRIB, J. (1990), The Spaniards in Rome. From Marius to Domitian, New York, Garland.

WeISGERBer, J.L. (1968), Die Namen der Ubier, Köln, Westdeutscher Verlag.

WodkTo, D.S. (2000), Monumenta linguarum hispanicarum vol. 5, 1. Wörterbuch der keltiberischen Inschriften, Wiesbaden, Ludwig Reichert.

ZeIDler, J. (2005), «Onomastic Studies on Some Roman Amici in Hispania», en CoşKUN, A. (ed.), Roms auswärtige Freunde in der späten Republik und im frühen Prinzipat, Göttingen, Göttingen Ruprecht Duehrkohp - Radicke, pp.175-200. 